\section{Fatores de risco para a mortalidade perinatal no Recife, Pernambuco, Brasil, 2003}

\author{
Risk factors for perinatal mortality in Recife, \\ Pernambuco State, Brazil, 2003
}

\footnotetext{
${ }^{1}$ Faculdade de Ciências Médicas, Universidade de Pernambuco, Recife, Brasil. 2 Diretoria Executiva de Epidemiologia, Secretaria de Saúde do Recife, Recife, Brasil.

Correspondência T. A. Aquino Departamento de Medicina Social, Faculdade de Ciências Médicas, Universidade de Pernambuco. Rua Amália Bernardino Sousa 710 , apto. 1503, Recife, $P E$

51021-150, Brasil. taa@recife.pe.gov.br
}

\begin{abstract}
The aim of this study was to identify and analyze risk factors for perinatal mortality in Recife, Pernambuco State, Brazil, in 2003, using a multilevel hierarchical model. In this case-control study, cases consisted of all perinatal deaths in 2003 in singleton infants with birth weight $\geq 500 \mathrm{~g}$ and without congenital malformations. The controls were live births from December 26, 2002, to December 31, 2003, with the same characteristics as the study group, but who survived $\geq 6$ days. By using record linkage techniques, 403 cases and 1,612 controls were obtained. All variables, when submitted jointly to multiple logistic regression, showed statistical significance in decreasing order of risk, as follows: prematurity $(O R=18.23)$, low birth weight $(O R=4.90)$, maternal age $\geq 35(\mathrm{OR}=1.97)$, delivery in public hospitals $(O R=1.93)$, and maternal schooling $<4$ years $(\mathrm{OR}=1.78)$.
\end{abstract}

Perinatal Mortality; Risk Factors; Case-control Studies
Terezinha de Almeida Aquino 1

Maria José Bezerra Guimarães 2

Sílvia Wanick Sarinho 1

Luiz Oscar Cardoso Ferreira 1

\section{Introdução}

A mortalidade perinatal, que compreende os óbitos fetais (com mais de 500g ou 22 semanas de gestação) e os neonatais precoces (ocorridos com até seis dias completos de vida), é reconhecida como um indicador sensível de avaliação da qualidade da assistência materna e neonatal 1 . Constitui um grave problema de saúde maternoinfantil, estando relacionada a uma complexa interação de fatores determinantes ${ }^{2}$. As gestantes, quando expostas a esses fatores de risco, podem apresentar complicações que comprometem também a sua saúde.

Anualmente, estimam-se mais de 7,6 milhões de mortes perinatais no mundo, das quais $57 \%$ são óbitos fetais e $98 \%$ ocorrem nos países em desenvolvimento. Dos 140 milhões de nascimentos anuais, no mundo, 4,3 milhões de fetos morrem depois da 22a semana de gestação e 3,3 milhões de recém-nascidos morrem antes de completar sete dias de vida ${ }^{3}$.

A mortalidade neonatal, especialmente na primeira semana de vida, representa o componente da mortalidade infantil de mais difícil redução. A partir dos anos 90 passou a ser o principal componente, no Brasil, em termos proporcionais, da mortalidade infantil, e não apresentou declínio considerável, mantendo-se com tendência a estabilidade em níveis elevados ${ }^{2}$. No país, as ações necessárias para o seu controle são 
ainda incipientes, demandando priorização nas políticas de saúde 1 .

A mortalidade perinatal resulta de uma complexa cadeia causal, em que determinantes proximais, como prematuridade e crescimento intra-uterino retardado (com ou sem baixo peso ao nascer), são desencadeados pelos determinantes intermediários e pelos determinantes distais 4 .

Os determinantes proximais da mortalidade perinatal estão relacionados com as variáveis biológicas referentes à mãe e ao recém-nascido e constituem as causas diretas dos óbitos perinatais 5 . O baixo peso ao nascer, considerado como aquele inferior a $2.500 \mathrm{~g}$ no momento do nascimento, representa o fator biológico que mais influencia a mortalidade perinatal 6 .

Os determinantes intermediários são capazes de interferir nos fatores de risco biológicos. São representados pela assistência pré e perinatal, tipo de parto, tipo de hospital, história reprodutiva, hábitos de vida e doenças maternas 4,5.

Entre os determinantes distais da mortalidade perinatal, os fatores sócio-econômicos são os mais importantes, por sua capacidade de influenciar alguns efeitos dos fatores biológicos e dificultar o acesso a uma assistência adequada à gestante durante o pré-natal e o nascimento da criança 4,5 .

Uma das limitações do estudo dos coeficientes de mortalidade perinatal deve-se à má qualidade dos registros dos óbitos em virtude da subnotificação e dos diferentes critérios utilizados para a classificação de morte ${ }^{7}$. O erro de classificação entre nascido vivo e nascido morto, considerado como clássico problema nas estimativas de mortalidade infantil, pode subestimar ou superestimar a mortalidade fetal ou a neonatal 8 .

Outra limitação importante do estudo da mortalidade perinatal refere-se ao registro da duração da gestação e peso ao nascer, dados freqüentemente omitidos na Declaração de Óbito (DO), comprometendo a definição precisa do período perinatal. As comparações temporais e espaciais também podem ser invalidadas por utilizarem diferentes conceitos de período perinatal, uma vez que as revisões anteriores à Classificação Estatística Internacional de Doenças e Problemas Relacionados à Saúde, 10a Revisão (CID-10) adotavam o critério de 28 semanas de gestação como limite inferior do período 9 .

Este estudo, ao enfocar os fatores de risco para a mortalidade perinatal, levou em consideração que os óbitos perinatais constituem um importante problema de saúde materno-infantil no país e, mais especificamente, no Recife, Estado do Pernambuco. Considerou-se, ainda, que tais óbitos apresentam fatores de risco potencialmente redutíveis, particularmente aqueles rela- cionados à atenção e à saúde, e que, no Brasil, existem poucos estudos que enfocam fatores de risco, nenhum referente ao estado pernambucano ou a seus municípios. Desse modo, este trabalho torna-se importante para uma melhor compreensão dos fatores determinantes da mortalidade perinatal no Recife e para o embasamento de políticas públicas de atenção à saúde materno-infantil, tais como a adoção de medidas mais apropriadas de atenção às gestantes, no pré-natal e na ocasião do parto, e aos recém-nascidos, de modo que se reduza a mortalidade fetal, neonatal e perinatal no município.

\section{Método}

O estudo foi realizado no Município do Recife, capital de Pernambuco, localizado no litoral da Região Nordeste do Brasil, ocupando uma área de $219 \mathrm{~km}^{2}$, totalmente urbano, com características ambientais diversificadas, onde há grandes desigualdades sociais 10 .

O desenho de estudo foi caso-controle, sendo considerados como caso todos os óbitos perinatais ocorridos em 2003 com peso ao nascer igual ou superior a $500 \mathrm{~g}$, de gravidez única de residentes na cidade do Recife, não-portadores de anencefalia. Como controles, consideraram-se os nascidos vivos entre 26 de dezembro de $2002 \mathrm{e}$ 31 de dezembro de 2003, que não evoluíram para o óbito até seis dias completos de vida, com as mesmas características dos casos.

O tamanho da população a ser estudada (283 óbitos e 1.132 controles) baseou-se na identificação de estudos brasileiros, a partir da década de 1990, sendo considerada a relação de 1 caso para 4 controles, nível de significância de $95 \%$ $(\alpha=0,05)$ e poder de $80 \%$ ( $(=20 \%)$. Com a finalidade de garantir maior margem de segurança, ao apresentar variáveis como o índice sintético de condição de vida, ainda não testado nesse tipo de análise, incluíram-se todos os 403 óbitos perinatais. Para a seleção dos controles, foi realizada uma amostra aleatória simples, tendo como população-alvo os 24.233 nascidos vivos que se adequavam aos critérios de seleção, obtendo-se, então 1.612 controles.

A seleção das variáveis foi realizada considerando-se os referenciais teóricos sobre os determinantes da mortalidade perinatal, a sua disponibilidade no Sistema de Informações sobre Nascidos Vivos (SINASC) e Sistema de Informações sobre Mortalidade (SIM) e o seu grau de preenchimento. O número de consultas ao pré-natal não foi estudado por não ser conhecido para os óbitos fetais, o mesmo ocorrendo com o índice de Apgar por este não se aplicar aos óbitos fetais. 
A variável dependente correspondeu ao óbito perinatal. As variáveis independentes contemplam os três níveis hierárquicos de determinação da mortalidade perinatal: proximal (peso ao nascer, idade gestacional, sexo do recém-nascido e idade da mãe); intermediário (tipo de hospital: Sistema Único de Saúde - SUS - e não SUS; e tipo de parto) e distal (escolaridade da mãe e condição de vida do bairro de residência). A condição de vida, indicador sintético construído por Guimarães 10, foi mensurado por meio de análise fatorial com base em indicadores de saneamento, educação e renda. Para o bairro de residência da população estudada, foi verificada qual a condição de vida respectiva.

Na categoria de estabelecimentos pertencentes ao SUS, incluíram-se as maternidades próprias/conveniadas, as instituições universitárias, filantrópicas e privadas conveniadas, além de três maternidades que, apesar de mantidas pelo poder público, oferecem acesso restrito a servidores públicos.

Os dados referentes aos casos e controles tiveram como fonte o SIM e o SINASC, operacionalizado pela Secretaria de Saúde do Recife.

Para estruturação do banco final de dados, a fim de permitir sua análise, foram utilizados diversos procedimentos, técnicas e programas de informática, conforme as etapas, especificadas na Figura 1.

Para validação dos dados, foram comparadas variáveis em comum, presentes nas DO e Declarações de Nascidos Vivos (DNV), como peso ao nascer, sexo do recém-nascido, idade da mãe, duração da gestação, tipo de parto e escolaridade da mãe. A discordância variou entre 1,2\% e 8,1\%, dependendo da variável. Quando a diferença no valor da variável era capaz de interferir na categorização do caso, conferiram-se os dados discordantes com os das vias originais das DO e DNV, para excluir possíveis erros de digitação e, se necessário, realizou-se consulta nas maternidades para verificação da variável correta, sendo, então, realizada a correção no banco de dados do estudo.

Para a análise multivariada, utilizou-se o método de regressão logística múltipla, a fim de determinar o efeito independente de cada variável sobre a mortalidade perinatal. A análise foi realizada, inicialmente, para cada conjunto de variáveis que pertenciam aos determinantes proximais, intermediários e distais, de acordo com o modelo hierarquizado de determinação; posteriormente, realizou-se a regressão logística de todas as variáveis em conjunto, introduzindo cada variável no modelo multivariado, de acordo com o valor do odds ratio (OR) em ordem decrescente.
Considerando que a variável resposta era dicotômica, estabeleceu-se que a não-ocorrência do evento (óbito perinatal) seria igual a "0" e a ocorrência do óbito igual a " 1 ". Para todas as variáveis de exposição, considerou-se “0” para as categorias de referência.

A regressão logística foi realizada por meio do programa SPSS 8.0 (SPSS Inc., Chicago, Estados Unidos), utilizando-se o procedimento forward stepwise (LR) não condicional, com níveis de significância de 5\% e 10\% para inclusão e exclusão de variáveis, respectivamente. Foram obtidos os OR ajustados com seus respectivos intervalos de confiança, considerando o valor de p significante a $5 \%$.

\section{Resultados}

Entre os óbitos perinatais incluídos no estudo houve predominância dos fetais, que representaram $57,8 \%$ das mortes. Considerando-se os nascimentos ocorridos em 2003 com as mesmas características observadas para os óbitos, encontrou-se um coeficiente de mortalidade perinatal de 16,6 por mil nascimentos, com risco de morte fetal (9,6 óbitos por mil nascimentos) superior ao encontrado no período neonatal precoce $(7,1$ óbitos por mil nascidos vivos).

$\mathrm{Na}$ análise multivariada para identificação dos fatores de risco proximais, o peso ao nascer menor que $2.500 \mathrm{~g}$ apresentou risco de morte 4,9 vezes maior do que o peso igual ou superior a 2.500g; a prematuridade (idade gestacional menor que 37 semanas) mostrou risco elevado de morte perinatal, em comparação com os nascidos a termo (OR = 19,90); a idade da mãe igual ou superior a 35 anos apresentou risco 1,84 vez maior que a idade da mãe inferior a 35 anos. O sexo masculino não mostrou associação com a mortalidade perinatal (Tabela 1).

Na identificação dos fatores de risco intermediários, para os nascidos em estabelecimentos de saúde participantes do SUS, o risco de morte perinatal foi 1,65 vez maior do que o dos que nasceram em hospitais que não faziam parte do SUS. O parto cesariano se apresentou com OR ajustado inferior a 1 (Tabela 2).

Quanto aos determinantes distais, a escolaridade da mãe - menos de quatro anos de estudo - apresentou-se como fator de risco para a mortalidade perinatal, sendo 2,09 vezes maior do que para os nascidos de mulheres com quatro ou mais anos de estudo. A condição de vida do bairro de residência, para os filhos de mães residentes no estrato de baixa e intermediária condição de vida, o risco de morte perinatal foi 1,45 vez maior do que entre aquelas que residiam no estrato de alta condição de vida (Tabela 3 ). 
Figura 1

Diagrama sobre a estruturação dos bancos.

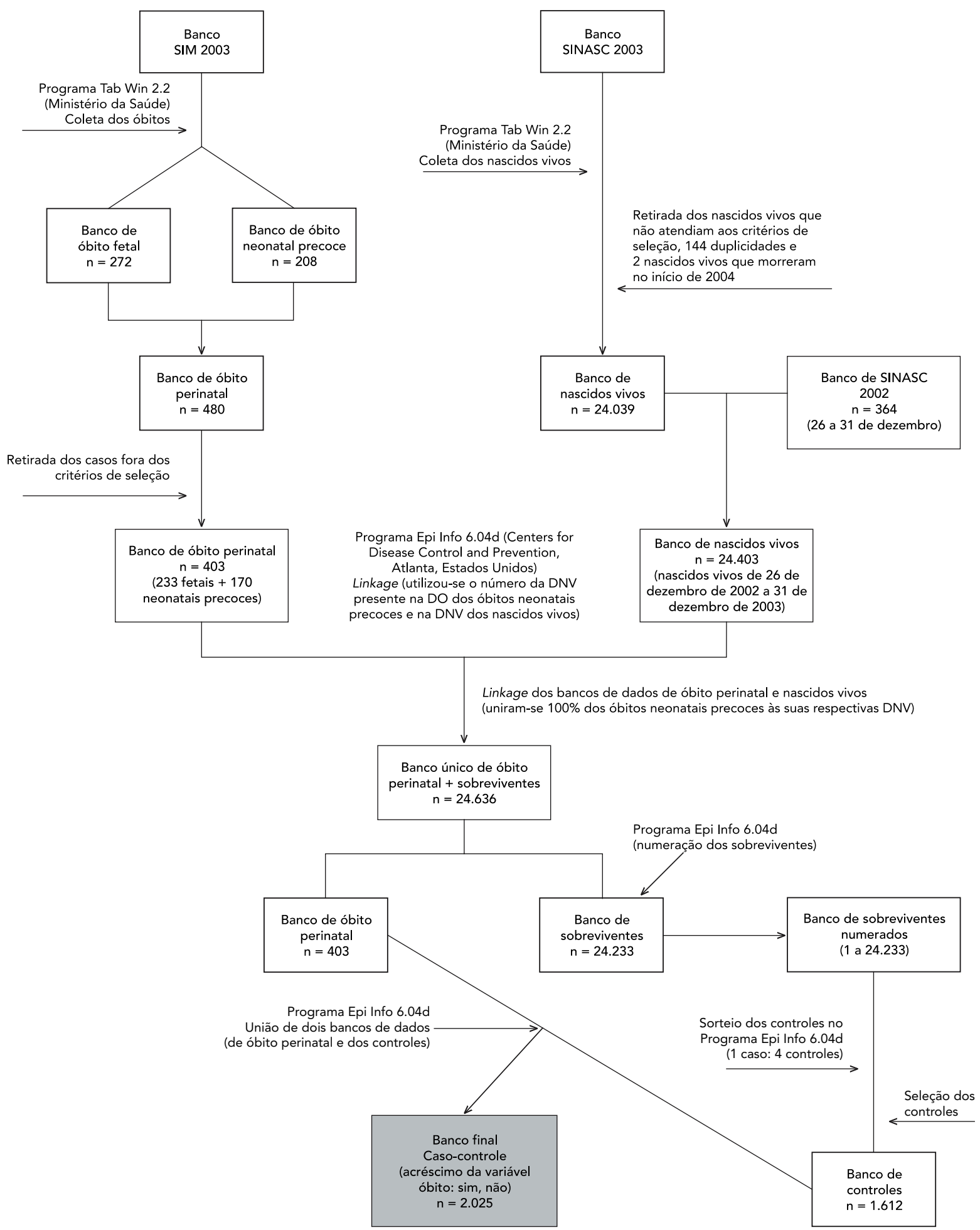

SIM: Sistema de Informações sobre Mortalidade; SINASC: Sistema de Informações sobre Nascidos Vivos; DO: Declaração de Óbito; DNV: Declaração de Nascidos Vivos. 
Fatores de risco ajustados para a mortalidade perinatal segundo determinantes proximais. Recife, Pernambuco, Brasil, 2003.

\begin{tabular}{|c|c|c|c|c|c|}
\hline Variáveis & Casos & Controles & OR ajustado & IC95\% & $\mathrm{p}$ \\
\hline \multicolumn{6}{|c|}{ Peso ao nascer (g) } \\
\hline$<2.500$ & 290 & 121 & 4,90 & $3,19-7,54$ & $<0,01$ \\
\hline$>2.500$ & 101 & 1.490 & 1,00 & & \\
\hline \multicolumn{6}{|c|}{ Duração da gestação (semanas) } \\
\hline$<37$ & 299 & 79 & 19,90 & $12,92-30,64$ & $<0,01$ \\
\hline$\geq 37$ & 92 & 1.532 & 1,00 & & \\
\hline \multicolumn{6}{|l|}{ Sexo } \\
\hline Masculino & 216 & 828 & 0,89 & $0,64-1,26$ & 0,53 \\
\hline Feminino & 183 & 784 & 1,00 & & \\
\hline \multicolumn{6}{|c|}{ Idade da mãe (anos) } \\
\hline$\geq 35$ & 53 & 117 & 1,84 & $1,07-3,17$ & $<0,05$ \\
\hline$<35$ & 342 & 1.495 & 1,00 & & \\
\hline
\end{tabular}

Tabela 2

Fatores de risco ajustados para a mortalidade perinatal segundo determinantes intermediários. Recife, Pernambuco, Brasil, 2003.

\begin{tabular}{lcccc}
\hline Variáveis & Casos & Controle & OR ajustado & IC95\% \\
\hline $\begin{array}{l}\text { Tipo de hospital } \\
\text { SUS }\end{array}$ & 353 & 1.248 & 2,39 & $1,68-3,40$ \\
Não SUS & 43 & 363 & 1,00 & $<0,01$ \\
Tipo de parto & & & & $0,34-0,56$ \\
Cesáreo & 109 & 747 & 0,43 & 1,00 \\
Vaginal & 291 & 865 & 0,01 \\
\hline
\end{tabular}

Tabela 3

Fatores de risco ajustados para a mortalidade perinatal segundo determinantes distais. Recife, Pernambuco, Brasil, 2003.

\begin{tabular}{lccc}
\hline Variável & Casos & Controles & OR ajustado \\
\hline $\begin{array}{l}\text { Escolaridade da mãe (anos) } \\
\quad 4\end{array}$ & 58 & 144 & 2,09 \\
$\quad \geq 4$ & 267 & 1.426 & 1,00 \\
$\begin{array}{l}\text { Condição de vida do bairro de residência } \\
\text { Baixa + intermediária }\end{array}$ & 351 & 1.334 & 1,45 \\
$\quad$ Alta & 52 & 277 & 1,00 \\
\hline
\end{tabular}


Todos esses fatores de risco foram submetidos, conjuntamente, à técnica da regressão logística múltipla. Como resultados, permaneceram com significância estatística a prematuridade $(\mathrm{OR}=18,23)$, que se apresentou como o fator mais fortemente associado à mortalidade perinatal, e, em ordem decrescente do risco, o baixo peso ao nascer $(\mathrm{OR}=4,90)$; a idade da mãe igual a ou maior que 35 anos $(\mathrm{OR}=1,97)$; o hospital participante do SUS $(\mathrm{OR}=1,93)$ e a escolaridade da mãe - menos de quatro anos de estudo $(\mathrm{OR}=$ 1,78) (Tabela 4).

\section{Discussão}

O aumento do percentual dos óbitos neonatais precoces, observado na última década no Brasil, pode ser explicado, segundo Carvalho \& Gomes 11, pelo contexto desfavorável de dificuldade de acesso aos serviços de saúde e pela iniqüidade e precariedade da assistência perinatal. Não se pode também desconsiderar o aumento da visibilidade dos óbitos neonatais, uma vez que muitos dos que eram contabilizados como natimortos, por serem considerados inviáveis, passaram a ser corretamente classificados como nascidos vivos, nos últimos anos, ocorrendo o registro do óbito 11 .

Para a redução da mortalidade perinatal, é importante melhorar a qualidade das informações das DO e fazer uso delas, a fim de subsidiar

Tabela 4

Regressão logística multivariada do conjunto de fatores de risco proximais, intermediários e distais para a mortalidade perinatal. Recife, Pernambuco, Brasil, 2003.

\begin{tabular}{|c|c|c|c|}
\hline Variáveis & OR ajustado & IC95\% & $p$ \\
\hline \multicolumn{4}{|c|}{ Peso ao nascer (g) } \\
\hline$<2.500$ & 4,90 & $3,10-7,72$ & $<0,01$ \\
\hline$\geq 2.500$ & 1,00 & & \\
\hline \multicolumn{4}{|c|}{ Duração da gestação (semanas) } \\
\hline$<37$ & 18,23 & $11,52-28,84$ & $<0,01$ \\
\hline$\geq 37$ & 1,00 & & \\
\hline \multicolumn{4}{|c|}{ Idade da mãe (anos) } \\
\hline$\geq 35$ & 1,97 & $1,10-3,52$ & 0,02 \\
\hline$<35$ & 1,00 & & \\
\hline \multicolumn{4}{|c|}{ Tipo de hospital } \\
\hline SUS & 1,93 & $1,15-3,22$ & 0,01 \\
\hline Não SUS & 1,00 & & \\
\hline \multicolumn{4}{|c|}{ Escolaridade da mãe (anos) } \\
\hline$<4$ & 1,78 & $1,08-2,92$ & 0,02 \\
\hline$\geq 4$ & 1,00 & & \\
\hline
\end{tabular}

análises, avaliar os serviços de saúde e organizar a rede assistencial para a gestante e o recémnascido.

O presente estudo realizou uma análise hierarquizada para identificar os fatores de risco para mortalidade perinatal segundo seus determinantes proximais, intermediários e distais, modelo passível de ser utilizado nos serviços de saúde.

Entre os determinantes proximais, mostraram-se associados ao óbito perinatal o baixo peso ao nascer, a prematuridade e a idade materna igual ou superior a 35 anos de idade. O sexo, analisado nesse grupo de determinantes, não se apresentou como fator de risco para a mortalidade perinatal. Resultados semelhantes foram encontrados no Recife e em Fortaleza (Estado do Ceará) em 1996 12,13.

A prematuridade e o baixo peso ao nascer apresentam importante associação com a sobrevivência fetal e neonatal precoce 14 . O baixo peso ao nascer e a duração da gestação não devem ser estudados como fatores de risco isolados, mas como mediadores, por intermédio dos quais atuam diversos determinantes, como a escolaridade materna, as condições sócio-econômicas, as características biológicas e os hábitos de vida da mãe, o acesso aos serviços de saúde durante a gestação, a qualidade desses serviços, entre outros 15 .

Especificamente no que se refere à mortalidade dos recém-nascidos muito prematuros, existem dificuldades bem reconhecidas para o estudo desses óbitos no Brasil, como a baixa confiabilidade dos dados da DO e a pobreza de informações detalhadas 11. No entanto, neste estudo, para validade dos dados, foram comparadas variáveis em comuns, presentes nas DO e DNV, como peso ao nascer, sexo do recém-nascido, idade da mãe, duração da gestação, tipo de parto e escolaridade da mãe.

$\mathrm{Na}$ presente pesquisa, a prematuridade, quando ajustada por outras variáveis do modelo multivariado, persistiu como um fator de risco importante para a mortalidade perinatal, sendo a variável que se mostrou mais fortemente associada ao óbito perinatal.

É contraditória a influência da idade materna na mortalidade perinatal. É importante ressaltar que, no que se refere à idade materna, os fatores biológicos podem ser menos relevantes do que determinados fatores sócio-econômicos, podendo, portanto, gerar distorções na avaliação desta variável no risco perinatal 16. Alguns estudos demonstram associação entre o risco de óbito perinatal e as idades maternas consideradas no extremo da vida reprodutiva, ou seja, inferiores a 20 e superiores a 34 anos 7,17. Porém, 
alguns autores não encontraram a mesma associação 5,18 .

Entre mães adolescentes, a mortalidade perinatal tem sido atribuída ao baixo peso ao nascer e à prematuridade. No entanto, sugere-se que esses fatores não sejam causas diretas ou determinantes independentes. $\mathrm{O}$ aspecto biológico não pode ser analisado de maneira isolada, visto que as condições psicossociais são de grande importância 19 . Além desses fatores, a falta de cuidados pré-natais, associados à pobreza e ao baixo nível de escolaridade, têm papel importante na cadeia causal de recém-nascidos com baixo peso ao nascer entre as adolescentes 20 .

É possível que a não-associação de morte perinatal com mães adolescentes, neste estudo, resulte do pequeno número de óbitos perinatais de nascidos de mães com menos de 15 anos, interferindo na análise estatística. Na literatura, as pesquisas não são unânimes em relação a essa associação. Em 1998, na cidade do Recife, encontrou-se um risco de morte para o componente neonatal de 1,54 vez, quando se compararam as mães adolescentes com as não adolescentes; porém, na análise multivariada, essa associação não se manteve 21 .

A idade materna igual ou superior a 35 anos apresentou-se associada à mortalidade perinatal no Recife. Resultados semelhantes foram encontrados na cidade de Pelotas (Estado do Rio Grande do Sul) ${ }^{22}$. Outros estudos não comprovaram essa associação, como o realizado na cidade do Recife em 1998, em relação ao componente da mortalidade neonatal 21 .

Tais achados podem estar associados com um maior número de anomalias congênitas encontradas nessa faixa etária, que aumentam progressivamente a cada ano de vida da mulher 16. É provável que as crianças morram antes do nascimento. Nesse estudo foi maior o contingente relativo aos óbitos fetais.

Quanto aos aspectos assistenciais que têm influência no risco de morte perinatal, no presente estudo, os nascimentos ocorridos nos estabelecimentos de saúde que fazem parte do SUS apresentaram risco maior de morte perinatal, mesmo quando houve ajuste pelo tipo de parto.

Resultado semelhante ao do presente estudo, no que diz respeito à evidência de associação do óbito neonatal/perinatal com a entidade mantenedora do hospital de nascimento, foi encontrado para o Município de Santo André (Estado de São Paulo) 23.

Inúmeras dificuldades relacionadas à garantia do acesso - iniqüidade em lugar de equidade, desorganização e fragmentação, em vez da regionalização e hierarquização do sistema, como também as freqüentes inadequações técnico- científicas na assistência - ainda se apresentam como desafios muito presentes para a gestão pública do atendimento perinatal no país ${ }^{11}$.

No presente estudo, o parto cesariano, semelhantemente aos achados de outros autores 21,23, apresentou-se com OR ajustado pelo tipo de hospital (SUS, não SUS) abaixo de 1. Porém, quando ajustado pelas demais variáveis (peso ao nascer, idade gestacional, tipo de hospital, idade materna, escolaridade da mãe e condição de vida do bairro de residência), foi excluído do modelo final por não apresentar significância estatística.

Desigualdades sócio-econômicas regionais em relação à mortalidade perinatal têm sido evidenciadas no Brasil, indicando que o maior risco de morte se relaciona com o nível sócio-econômico das mães. As diferenças nas condições de saúde ou no acesso aos serviços de saúde têm sido analisadas em função do nível sócio-econômico, seja este mensurado pela renda, educação, ocupação ou posição na hierarquia social 24

Como variáveis sócio-econômicas, foram estudadas a escolaridade materna e a condição de vida do bairro de residência da mãe. A baixa escolaridade materna e a baixa condição de vida do bairro de residência se apresentaram como fatores de risco para a mortalidade perinatal, porém, quando ajustada para as demais variáveis do estudo, a condição de vida do bairro de residência da mãe perdeu sua significância estatística.

A baixa escolaridade materna tem sido apontada como fator de risco importante para a mortalidade fetal e neonatal e, também, para os nascimentos com baixo peso 23,25 . A baixa instrução causa desinformação, constituindo ainda fator condicionante de menor interesse pelos cuidados com a saúde ou de maior dificuldade de acesso aos serviços de saúde, aspectos que podem ter um efeito importante na determinação da mortalidade perinatal.

Na região de Guaratinguetá (Vale do Paraíba Paulista, São Paulo), em 2001, observou-se maior comparecimento à consulta de pré-natal entre as gestantes com maior nível de escolaridade 25 . Já em Fortaleza, em 1996, o nível de escolaridade foi o segundo fator mais importante para a mortalidade fetal, mesmo após ajuste para a idade da mãe e a renda familiar 13 .

No presente trabalho, no modelo final, quando foram ajustadas todas as variáveis do estudo que apresentaram significância estatística, a análise multivariada revelou maior força de associação entre morte perinatal e prematuridade, seguido pelo baixo peso ao nascer. Outras variáveis, como idade da mãe superior a 34 anos, hospital de nascimento pertencente ao SUS e escolaridade materna, mantiveram-se como fatores de risco para a mortalidade perinatal. 
Os fatores de risco que estiveram mais fortemente associados à mortalidade perinatal foram a prematuridade e o baixo peso ao nascer, passíveis, em certo grau, de influências assistenciais e sócio-econômicas. Para a prevenção da ocorrência desses fatores, devem ser implementadas, além de medidas mais amplas, como melhorias na qualidade de vida da população (renda, escolaridade), medidas setoriais, como a garantia de realizar um pré-natal de

\section{Resumo}

Foi realizado estudo caso-controle com o objetivo de analisar os fatores de risco associados à mortalidade perinatal no Recife, Pernambuco, Brasil, 2003, de acordo com um modelo hierarquizado de determinantes proximais, intermediários e distais. Foram considerados casos os óbitos perinatais com peso ao nascer igual ou superior a 500g, de gravidez única e não portador de anencefalia. Os controles foram os nascidos vivos entre 26 de dezembro de 2002 e 31 de dezembro de 2003, que não evoluíram para o óbito até seis dias completos de vida, com as mesmas características dos casos. Com o linkage entre o banco de dados do Sistema de Informações sobre Nascidos Vivos e o de óbitos perinatais, obtiveram-se 403 casos e 1.612 controles. Após regressão logística múltipla, com a inclusão de variáveis dos três níveis de determinação, constituíram-se fatores de risco para mortalidade perinatal: a prematuridade $(O R=$ 18,23), o baixo peso ao nascer $(O R=4,90)$, a idade da mãe igual a ou maior que 35 anos (OR =1,97), o nascimento em hospitais participantes do Sistema Único de Saúde $(O R=1,93)$ e a escolaridade da mãe inferior a quatro anos de estudo $(O R=1,78)$.

Mortalidade Perinatal; Fatores de Risco; Estudos de Casos e Controles qualidade e um atendimento materno e neonatal em unidades hospitalares com melhores condições.

Diante dessas reflexões, reveste-se de fundamental importância a realização de outros estudos sobre saúde perinatal, utilizando diferentes enfoques e abordagens. Entre eles, encontra-se o estudo da mortalidade fetal, ainda pouco explorado no país, apesar de sua indiscutível importância para a saúde materno-infantil.

\section{Colaboradores}

T. A. Aquino participou da construção dos bancos de dados, análise dos dados, desenvolvimento da metodologia utilizada, análise estatística e redação do artigo, sendo responsável pela versão final. M. J. B. Guimarães contribuiu na análise dos dados, desenvolvimento da metodologia utilizada, análise estatística e redação do artigo. S. W. Sarinho colaborou na análise dos dados, no desenvolvimento da metodologia utilizada e redação do artigo. L. O. C. Ferreira participou do desenvolvimento da metodologia utilizada, análise estatística e revisão do artigo. 


\section{Referências}

1. Ministério da Saúde. Manual dos comitês de prevenção do óbito infantil e fetal. Brasília: Ministério da Saúde; 2004.

2. Lansky S, França E, Leal MC. Mortalidade perinatal e evitabilidade: revisão de literatura. Rev Saúde Pública 2002; 36:759-72.

3. Ungerer R. Situação perinatal no mundo. Tema (proj RADIS) 1999; (17):18-20.

4. Fonseca SC, Coutinho ESF. Pesquisa sobre mortalidade perinatal no Brasil: revisão da metodologia e dos resultados. Cad Saúde Pública 2004; 20 Suppl 1:S7-19.

5. Martins EF, Velásquez-Meléndez G. Determinantes da mortalidade neonatal a partir de uma coorte de nascidos vivos, Montes Claros, Minas Gerais, 19971999. Rev Bras Saúde Matern Infant 2004; 4:40512.

6. Bortman M. Factores de riesgo de bajo peso al nacer. Rev Panam Salud Pública 1998; 3:314-21.

7. Menezes AMB, Barros FC, Victora CG, Alves C, Rocha C, Albernaz E, et al. Mortalidade perinatal em duas coortes de base populacional no Sul do Brasil: tendências e diferenciais. Cad Saúde Pública 1996; 12 Suppl 1:S33-41.

8. Schramm JMA, Szwarcwald CL. Diferenciais nas taxas de mortalidade neonatal e natimortalidade hospitalares no Brasil: um estudo com base no Sistema de Informações Hospitalares do Sistema Único de Saúde (SIH/SUS). Cad Saúde Pública 2000; 16:1031-40.

9. Rede Integrada de Informações para a Saúde. Indicadores de mortalidade. http://tabnet.datasus. gov.br/cgi/idb2000/fqc05.htm (acessado em 16/ Jun/2004).

10. Guimarães MJB. Mortalidade infantil: uma análise das desigualdades intra-urbanas no Recife [Tese de Doutorado]. Rio de Janeiro: Fundação Oswaldo Cruz; 2003.

11. Carvalho M, Gomes MASM. A mortalidade do prematuro extremo em nosso meio: realidade e desafios. J Pediatr (Rio J) 2005; 81 Suppl 1:S111-8.

12. Coutinho SB. Mortalidade neonatal em cinco maternidades do Recife, 1994. Recife: Fundo das Nações Unidas para a Infância; 1996.

13. Rouquayrol MZ, Correia LL, Barbosa LMM, Xavier LGM, Oliveira JW, Fonseca W. Fatores de risco de natimortalidade em Fortaleza: um estudo casocontrole. J Pediatr (Rio J) 1996; 72:374-8.

14. Luginaah IN, Lee KS, Abernathy TJ, Sheehan D, Webster G. Trends and variations in perinatal mortality and low birthweight: the contribution of socio-economic factors. Can J Public Health 1999; 90:377-81.
15. Morais Neto OL, Barros MBA. Fatores de risco para mortalidade neonatal e pós-neonatal na Região Centro-Oeste do Brasil: linkage entre bancos de dados de nascidos vivos e óbitos infantis. Cad Saúde Pública 2000; 16:477-85.

16. Lorenzi DRS, Tanaka ACd'A, Bozzetti MC, Ribas FE, Weissheimer L. A natimortalidade como indicador de saúde perinatal. Cad Saúde Pública 2001; 17:141-6.

17. Aquino MMA, Cecatti JG, Mariani Neto C. Risk factors associated to fetal death. São Paulo Med J 1998; 116:1852-7.

18. Grupo Colaborativo de Estudos Perinatais. Fatores perinatais relacionados com a morbidade e a mortalidade de recém-nascidos pertencentes a nove unidades neonatais do Município de São Paulo. J Pediatr (Rio J) 1996; 72:379-87.

19. Mariotoni GGB, Barros Filho AA. A gravidez na adolescência é fator de risco para o baixo peso ao nascer? J Pediatr (Rio J) 1998; 74:107-13.

20. Gama SGN, Szwarcwald CL, Leal MC, Theme Filha MM. Gravidez na adolescência como fator de risco para baixo peso ao nascer no Município do Rio de Janeiro, 1996 a 1998. Rev Saúde Pública 2001; 35:74-80.

21. Sarinho SW. Mortalidade neonatal na cidade do Recife: um estudo caso controle [Tese de Doutorado]. Recife: Centro de Ciências da Saúde, Universidade Federal de Pernambuco; 1998.

22. Menezes AMB, Barros FC, Victora CG, Tomasi E, Halpern R, Oliveira ALB. Fatores de risco para mortalidade perinatal em Pelotas, RS, 1993. Rev Saúde Pública 1998; 32:209-16.

23. Almeida MF. Mortalidade neonatal em Santo André [Tese de Doutorado]. São Paulo: Faculdade de Saúde Pública, Universidade de São Paulo; 1994.

24. Andrade CLT, Szwarcwald CL, Gama SGN, Leal MC. Desigualdades sócio-econômicas do baixo peso ao nascer e da mortalidade perinatal no Município do Rio de Janeiro, 2001. Cad Saúde Pública 2004; 20 Suppl 1: S44-51.

25. Haidar FH, Oliveira UF, Nascimento LFC. Escolaridade materna: correlação com os indicadores obstétricos. Cad Saúde Pública 2001; 17:1025-9.

Recebido em 14/Jul/2006

Versão final reapresentada em 20/Mar/2007

Aprovado em 22/Mai/2007 